\title{
Research and Realization of Electronic Measuring Instrument Based on Virtual Technology \\ Xiaolei Wang ${ }^{1,{ }^{*} a}$, Wei Song ${ }^{2, b}$ \\ ${ }^{1}$ Liren College, Yanshan University, Hebei Qinhuangdao 066004, China; \\ ${ }^{2}$ Qinhuangdao Power Supply Company, State Grid Jibei Electric Power Company Limited, Qinhuangdao 066000, China. \\ *Corresponding author Email: agreatlife2018@163.com, ${ }^{b}$ thegreatnessoflife@163.com
}

Keywords: virtual instrument, experimental teaching, LabVIEW, sound card, voltage calibration.

\begin{abstract}
This thesis focuses on electronic experiment teaching how to use the virtual instrument in-depth research, design virtual instrument based on the computer sound card. Virtual oscilloscope based on the use of producers and consumers of data collection mode, which solve the data acquisition and data processing simultaneously prone to data loss problems. This paper describes in detail how to set up the sound management software and sound card voltage calibration method. The virtual function signal generator and virtual oscilloscope were conducted a comprehensive test to verify the practicality of these two virtual instrument.
\end{abstract}

\section{Introduction}

Experiment is an important part of higher education practice. Electronic laboratories require a wide variety of instruments, which require a large amount of money. The concept of "software is instrument" is put forward by American National Instrument Co., Ltd., which is the core of virtual instrument technology. The use of virtual instrument technology can solve the problem of lack of laboratory equipment and lack of funds. Virtual instrument technology can be used to create remote electronic laboratories [1]. In this paper, a virtual instrument based on computer sound card is designed and tested [2].

\section{Design of virtual instrument}

Now use the sound card to achieve a lot of function signal generator articles, so this article is no longer described in detail.

Virtual oscilloscope data acquisition speed is greater than the data processing speed, will cause the loss of data acquisition, and then cause the program to run wrong. In order to solve the above problem, the virtual oscilloscope uses the framework of the producer and the consumer, in which the producer represents the data collection and the consumer represents the data processing. As shown in Figure 1. This architecture divides data collection and data processing into two loops, and executes both loops simultaneously. The data transfer between the producer and the consumer is achieved through a queue, which has a first-in first-out feature.

The virtual oscilloscope relies on the trigger function to synchronize the display waveform. The SLOPE function in the LabVIEW dual-channel oscilloscope example enables this functionality. 


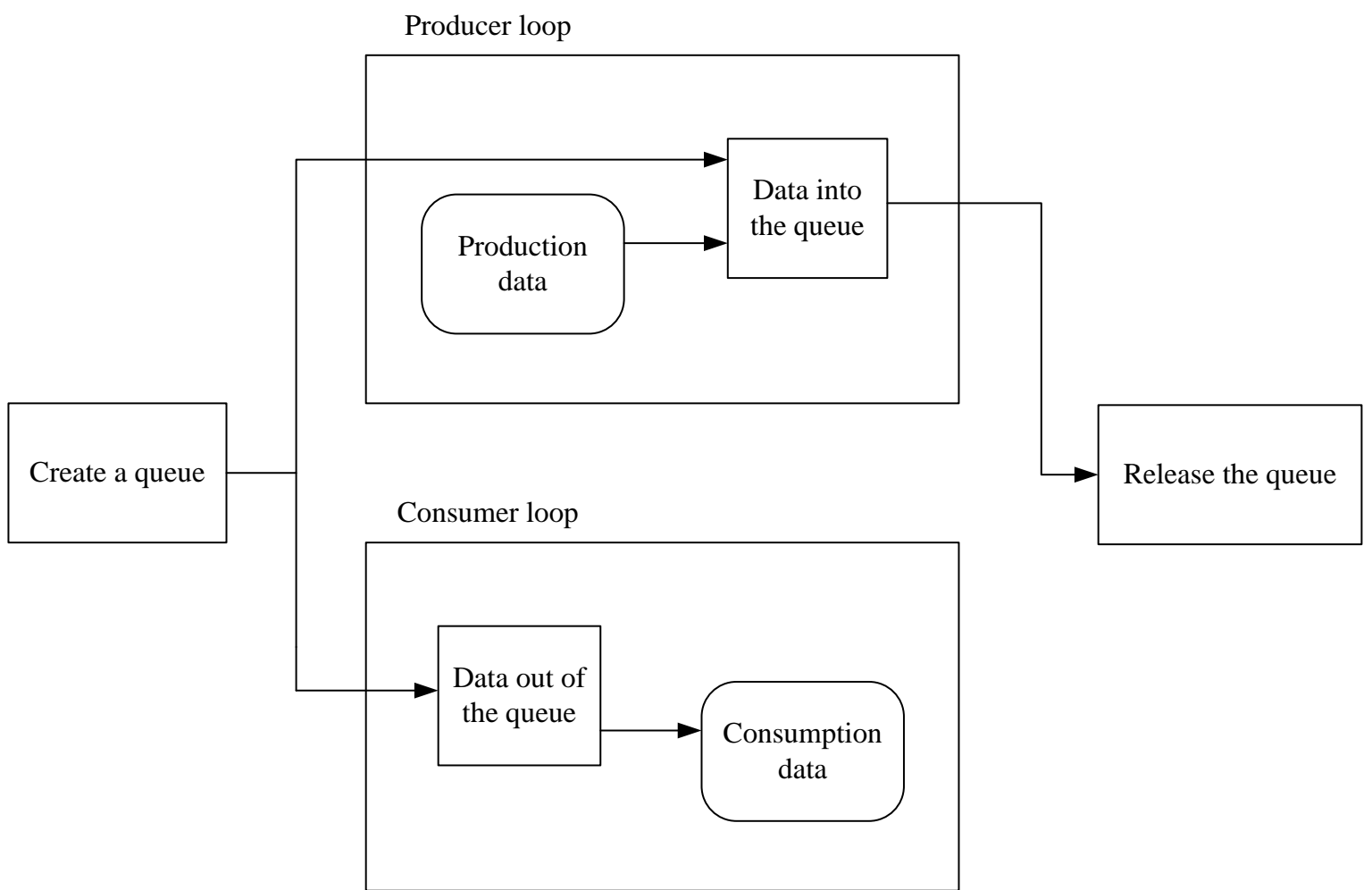

Fig. 1 Based on the data collection framework of producers and consumers

The front panel of the virtual oscilloscope is shown in Figure 2. The user interface of the virtual oscilloscope is similar to that of the traditional instrument, but the virtual oscilloscope data analysis function is far ahead of the traditional instrument. The virtual oscilloscope uses the statistical analysis function to calculate the peak, valley and peak-to-peak value of the signal. The virtual oscilloscope uses the spectrum measurement function to analyze the fast Fourier of the signal. The virtual oscilloscope uses the spectrum measurement function to analyze the frequency, amplitude and phase of the acquisition signal [3].

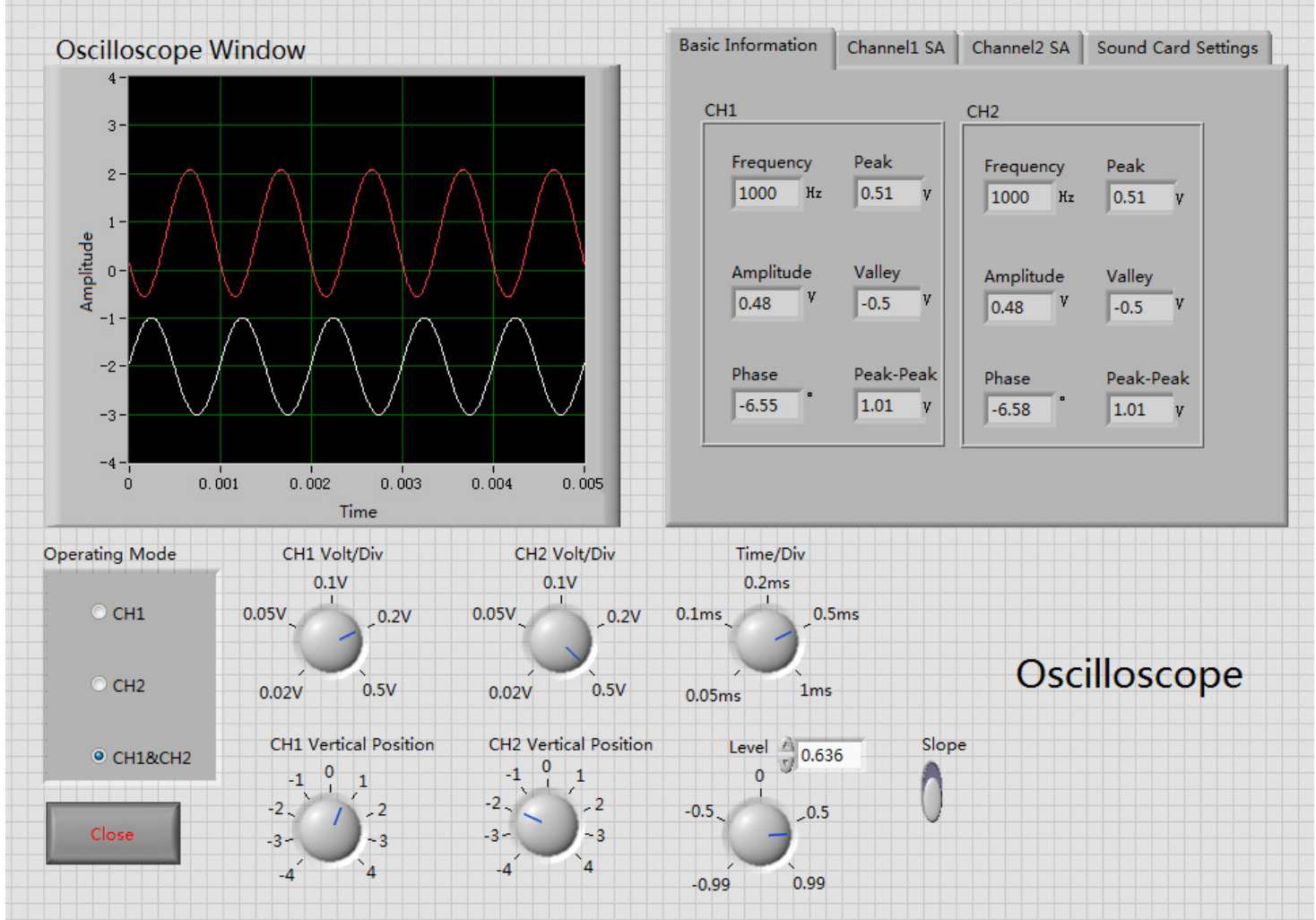

Fig. 2 The front panel of the virtual oscilloscope 


\section{Practical test}

Figure 3 from the left to the right are the Lotus head to BNC female adapter and $3.5 \mathrm{~mm}$ headphones to double the lotus mother's sub-two audio adapter cable. Through the above wiring, the sound card's Line In and Line Out interface is converted to the instrument commonly used BNC interface.

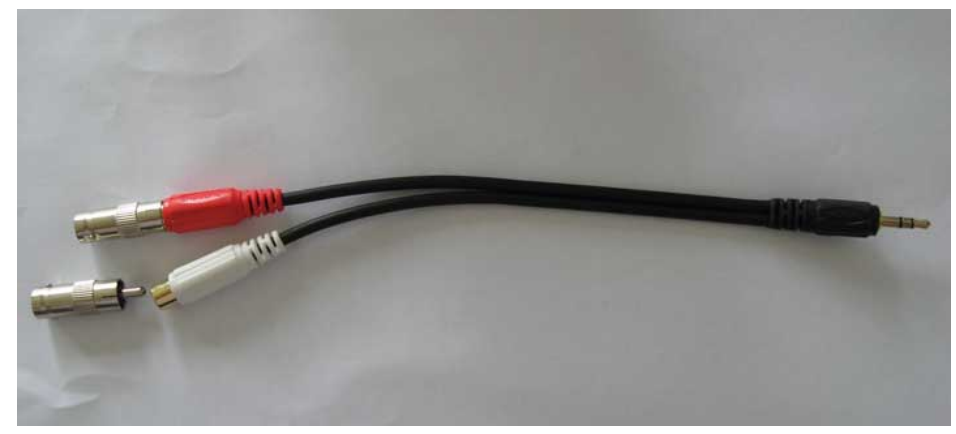

Fig. 3 Transfer of sound card interface

Sound card sound manager software how to set and voltage calibration, as shown in figure 4 . Open the sound manager, the environment in the audio interface to select no, turn off the equalizer. The replay knob of the mixer can calibrate the output voltage of the virtual signal generator. Turn off the other options on the right side of the screen, leaving only the waveform and Front. Recording knob can calibrate the acquisition voltage of virtual oscilloscope. Close other options on the right side of the recording, leaving only the line volume.

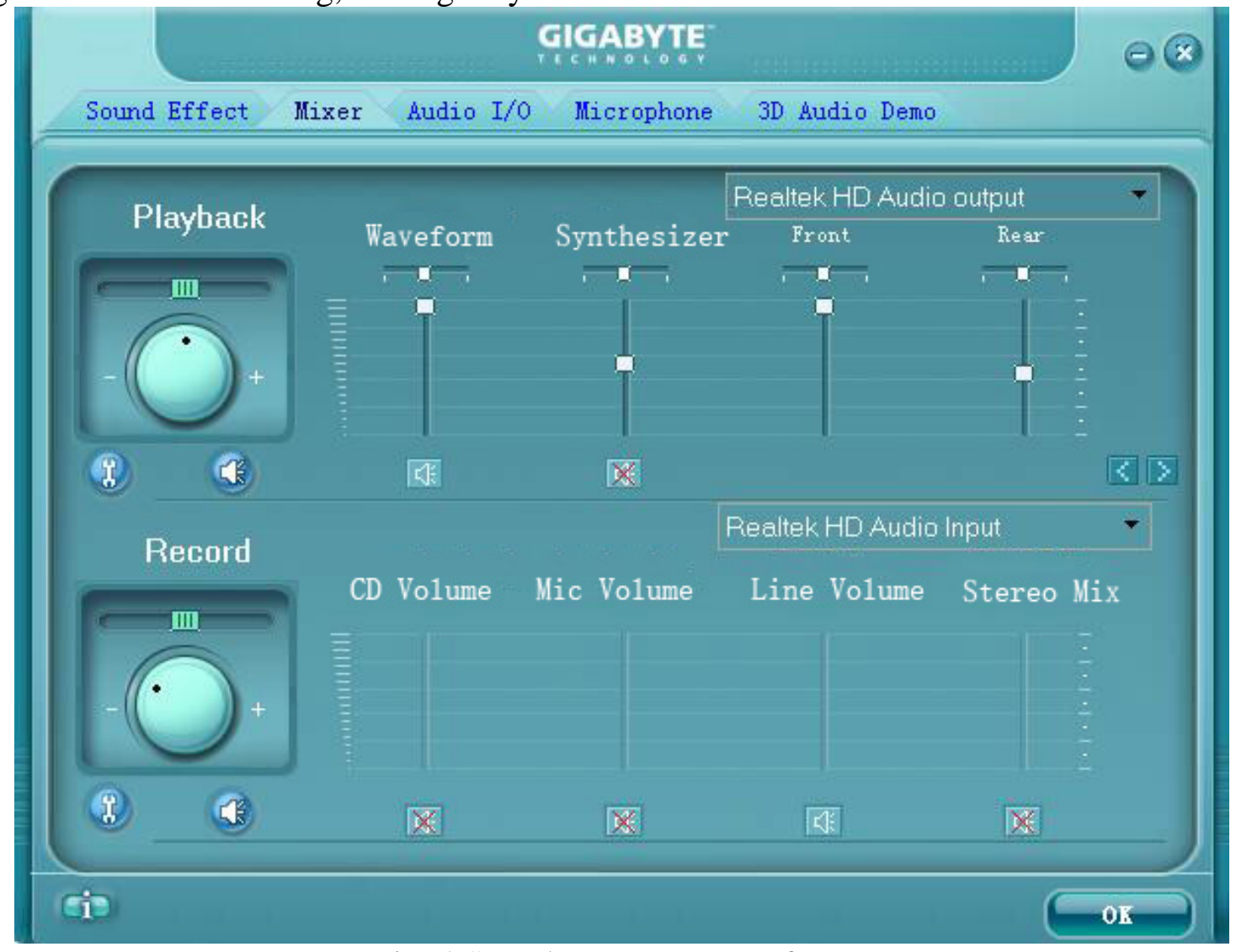

Fig. 4 Sound management software

Figure 5, 6, 7 are the virtual function signal generator output a variety of signals; Figure 8 is a virtual oscilloscope measuring $20 \mathrm{mV}$ small signal. 


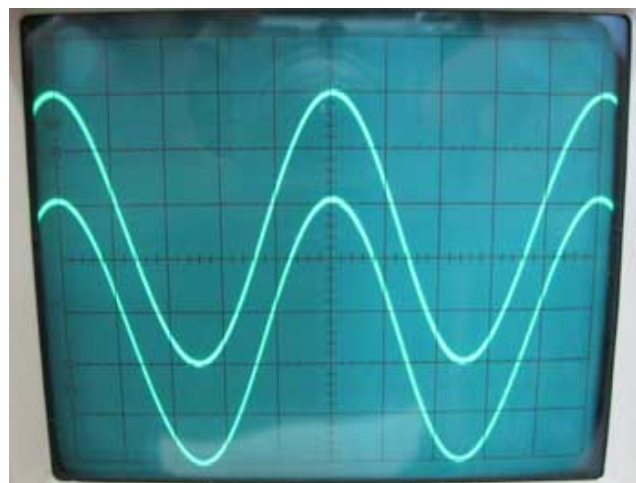

Fig. 5 Frequency 1kHz, peak-to-peak 1V sinusoidal signal

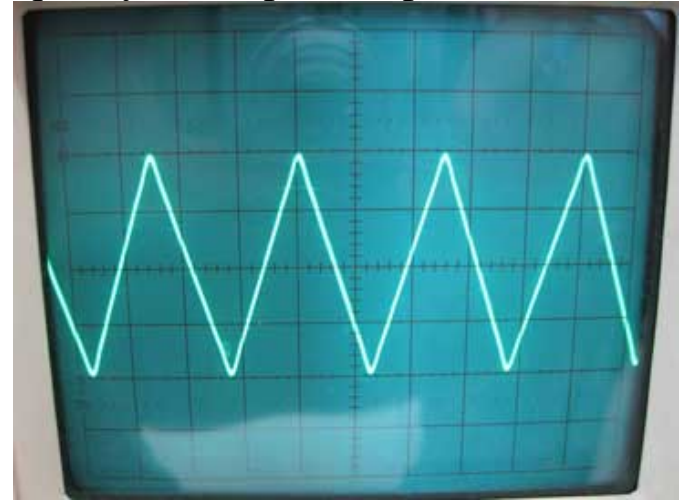

Fig. 6 Frequency 2kHz, peak-to-peak 2V triangular wave signal

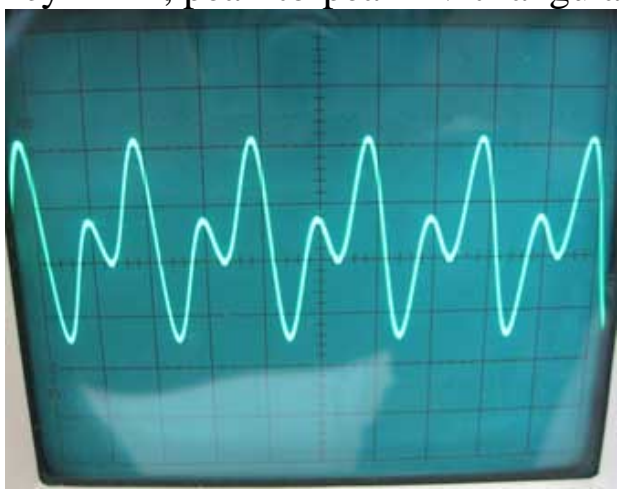

Fig. 7 Custom formula signal

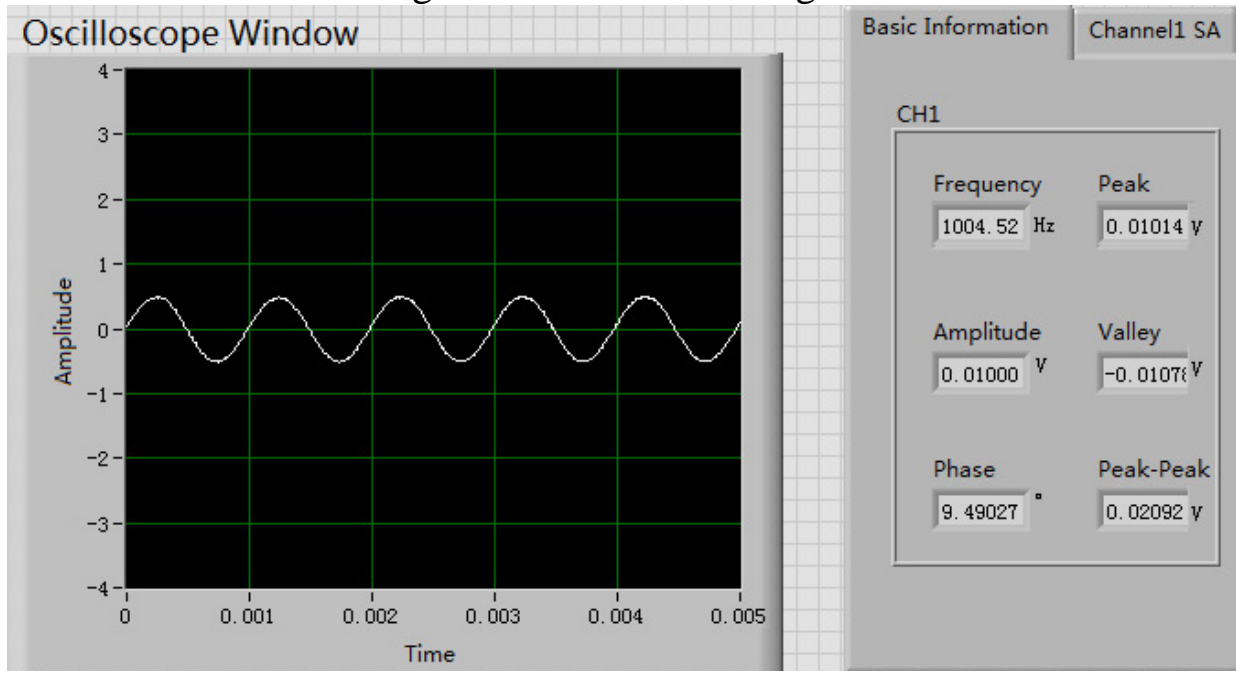

Fig. 8 Frequency $1 \mathrm{kHz}$, peak and peak $20 \mathrm{mV}$ sinusoidal signal

The circuit experiment has the experiment of observing the relationship between voltage and current of the capacitor. Virtual function signal generator output frequency $1 \mathrm{kHz}$, peak value of $1.5 \mathrm{~V}$ sine signal. The virtual oscilloscope channel 1 measures the voltage waveform at both ends of the capacitor (the larger one); the virtual oscilloscope channel 2 measures the current waveform of 
the current flowing through the capacitor. As shown in Figure 9, the capacitor voltage and current phase relationship is the current lead voltage of $90^{\circ}$.

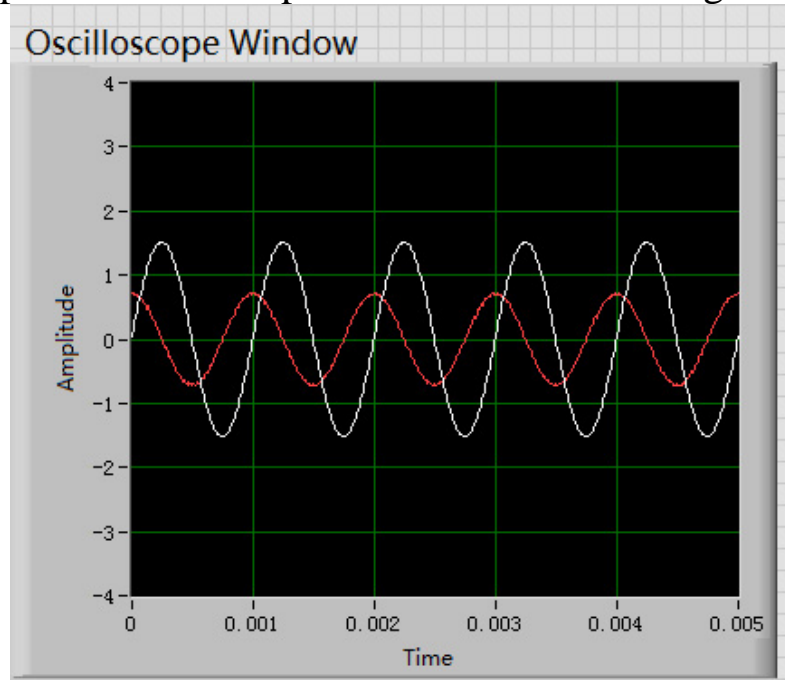

Basic Information Channel1 SA $\mid$ Channel2 SA Sound Card Settings

$\mathrm{CH} 1$

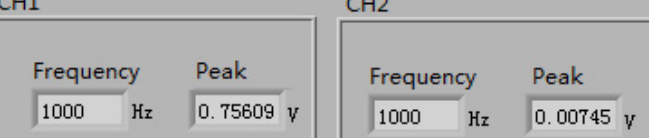

Amplitude Valley

0.75607 v -0.7562 y

\begin{tabular}{ll} 
Amolitude & Vallev \\
\hline 0.00711 y & $-0.0074 \mathrm{v}$
\end{tabular}

$\frac{\text { Phase }}{103.175} \cdot \frac{\text { Peak-Peak }}{1.51236 \mathrm{v}}$

Phase Difference

$-90$

Fig. 9 Capacitance voltage and current phase relationship

\section{Summary}

In this paper, a virtual signal generator and virtual oscilloscope are developed by using LabVIEW software. Students can easily use the virtual instrument to complete the electronic experiment. Compared with the traditional electronic laboratory, the virtual instrument can expand the new function and reduce the construction cost.

\section{Acknowledgments}

This work was supported by the Qinhuangdao city science and technology research and development plan program under Grant Nos. 201502A021

\section{References}

[1]. Bozidar Popovic, Natasa Popovic, Danijel Mijic, et al. Remote control of laboratory equipment for basic electronics courses: A LabVIEW-based implementation. Computer Applications in Engineering Education. Vol. 21 (2013) No. 1, p. 110-120.

[2]. Jan Saliga, Lin $\mu \mathrm{s}$ Michaeli. Software for metrological characterisation of PC sound cards. Computer Standards \& Interfaces. Vol. 25 (2003) No. 1, p. 45-55.

[3]. Ping Gong, Wei Zhou. Design and Implementation of Multifunctional Virtual Oscilloscope Using USB Data-Acquisition Card. Procedia Engineering. Vol. 29 (2012), p. 3245-3249. 